\section{Neurofibromatosis type 1 associated with coarctation of the abdominal aorta}

Reema K. AIEssa, ${ }^{1}$

Mohammed I. AlJasser ${ }^{1,2}$

${ }^{1}$ College of Medicine, ${ }^{2}$ Division of

Dermatology, King Saud bin Abdulaziz

University for Health Sciences, Riyadh, Saudi Arabia

\section{To the Editor}

Neurofibromatosis type 1 (NF1), is an autosomal dominant disease due to mutation in NF1 gene, affecting one in 3000 persons. ${ }^{1} \mathrm{NF} 1$ vasculopathy is a rare complication of NF1 with a frequency ranging from 0.4-6.4\%. ${ }^{1}$ It includes aneurysms, arterial stenosis, aorta coarctation, and arteriovenous malformations and it is highly associated with premature death. Coarctation of the abdominal aorta is a rare complication of NF1 in which Midaortic syndrome has an occurrence rate of $<10 \%$ among NF1 patients. ${ }^{2}$ We report a case of a 25 -years-old male with NF1 and coarctation of the

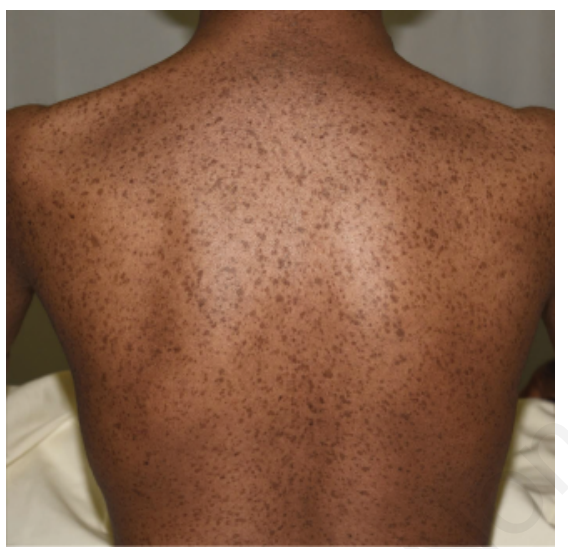

Figure 1. Multiple generalized dark brown macules over the back.

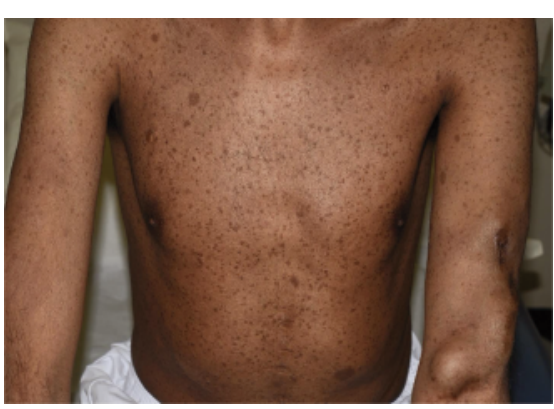

Figure 2. Generalized dark brown macules and several scattered café au lait macules over chest, abdomen, and left arm. Note arteriovenous fistula on left arm. abdominal aorta.

A 25-year-old Saudi male presented to the dermatology clinic with asymptomatic generalized brown spots since childhood. These spots continued to increase in size and number with time, but the cause was never investigated. No family members have similar skin lesions. Cutaneous examination revealed numerous generalized dark brown macules (Figure 1). In addition, he had several scattered café au lait macules (Figure 2). His past medical history includes hypertension, renal artery stenosis, and abdominal aorta stenosis. Left nephrectomy was performed in 2000 due to renal artery stenosis. He has been on hemodialysis due to declining renal function since 2011. CT angiogram showed significant focal stenosis of the abdominal aorta inferior to the level of the superior mesenteric artery (Figure 3). Furthermore, he had stenosis of several other abdominal arteries including right renal artery, celiac artery, and superior mesenteric artery. Whole exome sequencing on a peripheral blood sample identified a heterozygous mutation in the NF1 gene. Based on the above findings, a final diagnosis of NF1 associated with coarctation of the abdominal aorta was made.

Coarctation of the aorta is a rare complication in patients with NF1. One large study showed that only 5 patients had coarctation of the aorta among 2322 patients with NF1. ${ }^{3}$ Oderich et al. ${ }^{1}$ reviewed the literature from 1957 to 2005 and found

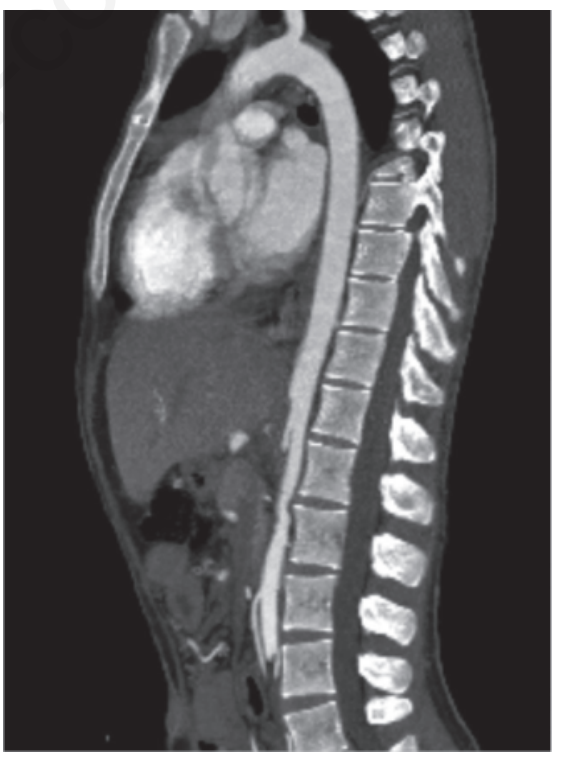

Figure 3. CT angiogram showing significant focal stenosis of the abdominal aorta inferior to the level of the superior mesenteric artery.
Correspondence: Reema Khalid Alessa, College of Medicine (COM), King Saud bin Abdulaziz University for Health Sciences (KSAU-HS), P.O. Box 3660, Riyadh 11481, Saudi Arabia

Tel.: +966.11.4299999 - Fax: +966.11.4299999 E-mail: r.k.alessa1@gmail.com

Key words: neurofibromatosis; NF1; abdominal aorta; renal artery.

Contributions: The authors contributed equally.

Conflict of interest: The authors declare no potential conflict of interest.

Funding: None.

Availability of data and material: Data and materials are available by the authors.

Please cite this article as: AlEssa RK, Al Jasser MI. Neurofibromatosis type 1 associated with coarctation of the abdominal aorta. Dermatol Rep 2022;14:9367.

Received for publication: 3 September 2021 Accepted for publication: 12 September 2021.

This work is licensed under a Creative Commons Attribution-NonCommercial 4.0 International License (CC BY-NC 4.0).

${ }^{\circ}$ Copyright: the Author(s), 2022

Licensee PAGEPress, Italy

Dermatology Reports 2022; 14:9367

doi:10.4081/dr.2022.9367

Publisher's note: All claims expressed in this article are solely those of the authors and do not necessarily represent those of their affiliated organizations, or those of the publisher, the editors and the reviewers. Any product that may be evaluated in this article or claim that may be made by its manufacturer is not guaranteed or endorsed by the publisher.

that 237 patients with NF1 had associated vascular abnormalities. Renal artery lesions were the most prevalent (41\%) while abdominal aorta coarctation or aneurysm were found in $12 \%$ of patients. The pathogenesis is unknown; however, there is role of neurofibromin deficiency in producing endothelial dysfunction. ${ }^{1}$ Several case reports demonstrated the association between abdomen aorta coarctation and NF1.2,4,5 Our patient developed end stage renal disease and unilateral nephrectomy at young age due to the associated coarctation of the abdominal aorta. We believed that early detection and frequent evaluation of vascular involvement are essential to prevent end organ complications and for timely intervention of vascular reconstruction. 


\section{References}

1. Oderich GS, Sullivan TM, Bower TC, et al. Vascular abnormalities in patients with neurofibromatosis syndrome type I: clinical spectrum, management, and results. J Vasc Surg 2007;46:475-84.

2. Veean S, Thakkar N, Gupta S,
Keshavamurthy J. A case of coarctation of the abdominal aorta and renal artery stenosis due to neurofibromatosis type 1. Postgrad Med J 2017;93:235-6.

3. Lin $\mathrm{AE}$, Birch $\mathrm{PH}$, Korf $\mathrm{BR}$, et al. Cardiovascular malformations and other cardiovascular abnormalities in neurofibromatosis 1. Am J Med Genet 2000;95:108-17.
4. Friedman JM, Arbiser J, Epstein JA, et al. Cardiovascular disease in neurofibromatosis 1: report of the NF1 Cardiovascular Task Force. Genet Med 2002;4:105-11.

5. Kurien A, John PR, Milford DV. Hypertension secondary to progressive vascular neurofibromatosis. Arch Dis Child 1997;76:454-5. 